\title{
Intra-individual variation of basal metabolic rate and the influence of daily habitual physical activity before testing
}

\author{
Mirjam P. E. Adriaens*, Paul F. M. Schoffelen and Klaas R. Westerterp \\ Department of Human Biology, Maastricht University, PO Box 616, 6200 MD Maastricht, The Netherlands
}

(Received 15 July 2002 - Revised 22 January 2003 - Accepted 23 March 2003)

\begin{abstract}
The present study determined the intra-individual variation of BMR measurements, using a standard out-patient protocol, with the subjects transporting themselves to the laboratory for the BMR measurements after spending the night at home. The effect of a non-fasting state and variation in daily habitual physical activity the day before testing was evaluated. Eight male and eleven female subjects participated in three BMR measurements with 2-week intervals. Physical activity was estimated with a tri-axial accelerometer for movement registration, during the $3 \mathrm{~d}$ before each BMR measurement. There were no significant differences in estimated BMR (ANOVA repeated measures, $P=0.88$ ) and in physical activity (ANOVA repeated measures, $P=0.21$ ). Mean within-subject CV in BMR was found to be 3.3 (SD 2.1$) \%$, ranging from 0.4 to $7.2 \%$. Differences between BMR measurements could not be explained by differences in physical activity the day before; however the mean within-subject CV in BMR changed from 5.7 to $5.2 \%$ after correcting for within-machine variability and from 5.2 to $3.3 \%$ after excluding five measurements because of non-compliance to the protocol including fasting. In conclusion, BMR values measured with a standard out-patient protocol are sufficiently reproducible for most practical purposes despite the within-subject variability in physical activity the day before the measurement. For this purpose, however, non-fasting subjects must be excluded and a regular function check of the ventilated-hood system is recommendable.
\end{abstract}

Reproducibility: Calorimetry: Body composition: Accelerometer

BMR is the main component of average daily metabolic rate. It is defined as the daily rate of energy metabolism an individual needs to maintain and preserve the integrity of vital functions. A measurement of BMR must meet certain conditions. The subject must be awake and the measurement must be performed in a thermoneutral environment to avoid heat production or heat loss for maintenance of body temperature. Furthermore the subject must be in a fasted state (absence of diet-induced thermogenesis) and in rest (absence of activity-induced energy expenditure)

Diet-induced thermogenesis is an increase in energy expenditure (EE) above BMR after eating. A $10-12 \mathrm{~h}$ fast before BMR measurements is the accepted procedure followed by investigators to eliminate the thermic effect of food on basal EE. However, the time interval required to eliminate any residual effect of physical activity on BMR has not yet been described in a similar way. Some studies have observed that moderate-intensity physical activity elevates metabolic rate for only a few minutes to a few hours (Bahr et al. 1992; Melby et al. 1993; Smith \& McNaughton, 1993; Quinn \& Vroman, 1994; Almuzaini et al. 1998); others observed an elevated BMR for a period of up to 24 h (Maehlum et al. 1986; Weststrate \& Hautvast, 1990; Herring et al. 1992; Williamson \& Kirwan, 1997; Borsheim et al. 1998; Osterberg \& Melby, 2000; Melanson et al. 2002). Finally, some studies did not observe any effect of physical activity including high-intensity exercise (Freedman-Akabas et al. 1985; Weststrate et al. 1990).

To perform an accurate measurement of BMR, subjects usually stay overnight in a hospital or research centre while nutritional intake and physical activity are strictly controlled, therefore assuring the absence of diet- and activity-induced thermogenesis. However, this method is expensive, time-consuming and inconvenient for both subjects and researchers. Additionally the aim of most studies is not just an accurate estimation of BMR but rather to detect changes in BMR due to an intervention. Therefore many studies use an out-patient protocol, which means that the subjects transport themselves to the laboratory for the BMR measurement after spending the night at home. Turley et al. (1993), Goran \& Nagy (1996) and Figueroa-Colon et al. (1996) compared the results of the out-patient protocol with the in-patient protocol. Goran \& Nagy (1996) found higher BMR values with the out-patient protocol (Goran \& Nagy, 1996), while Turley et al. (1993) 
and Figueroa-Colon et al. (1996) did not find significant differences. Turley et al. (1993) and Goran \& Nagy (1996) concluded that an in-patient protocol is the best for an accurate measurement of BMR. However, the change in BMR due to an intervention can be detected by using either an in-patient or an out-patient protocol (Turley et al. 1993; Goran \& Nagy, 1996). For this application a high reproducibility is especially important.

The aim of the present study was to determine the reproducibility or intra-individual variability of BMR after three repeated measurements, using an out-patient protocol. The disadvantage of an out-patient protocol is the impossibility to supervise the fasting state and the physical activity before the measurement. Therefore the present study planned to estimate the influence of variability in daily habitual physical activity and of non-compliance to the protocol including fasting on the reproducibility of BMR measurements. To a lesser degree, the reproducibility of BMR measurements is, for both an in-patient and an out-patient protocol, influenced by the within-machine variability of the ventilated-hood system. Consequently, the present study also focuses on the effect of the within-machine variability. Additionally, the goal of the study was to assess the inter-individual variability of BMR and to explain differences between subjects by differences in physical characteristics.

\section{Methods}

\section{Subjects}

Nineteen volunteers (eleven females and eight males, mean age 23.6 (SD 7.5) years) participated in the study. Criteria for subject selection were good health (with special attention given to the absence of metabolic diseases), and the absence of any use of medication that could have affected metabolic rate and weight stability during the last 6 months $( \pm 3 \mathrm{~kg})$. Also, the subjects were not consuming special diets or participating in exercise programmes. Subjects were informed of the procedures before they gave their consent to participate and the Medical Ethical Committee of the Maastricht University approved the protocol of the study.

Physical characteristics of the subjects are presented in Table 1.

\section{Experimental design}

At 1 week before the first BMR measurement a single assessment of body mass was performed. BMR was

Table 1. Physical characteristics of the subjects

\begin{tabular}{lccccc}
\hline & \multicolumn{2}{c}{ Male $(n 8)$} & & \multicolumn{2}{c}{ Female $(n 11)$} \\
\cline { 2 - 3 } & Mean & SD & & Mean & SD \\
\hline Age (years) & 23 & 5 & & 24 & 9 \\
Body mass $(\mathrm{kg})$ & 78.6 & 14.3 & & 61.7 & 7.2 \\
Height $(\mathrm{m})$ & 1.86 & 0.1 & & 1.73 & 0.1 \\
Fat mass $(\mathrm{kg})$ & 14.4 & 5.9 & & 18.2 & 5.9 \\
Fat-free mass $(\mathrm{kg})$ & 64.3 & 10.3 & & 43.6 & 3.7 \\
\hline
\end{tabular}

measured three times at 2 -week intervals using a ventilated-hood system. The measurements were repeated at the same time and day of the week for each subject. During the $3 \mathrm{~d}$ before each measurement physical activity was estimated. The day of the measurement started with the BMR assessment, followed by an estimation of body weight. An underwater weighing for measuring body density took place at the end of the first and the second day of testing.

The function of the ventilated-hood system was checked with methanol burning, alternately before or after each BMR measurement.

\section{Basal metabolic rate}

Subjects were instructed to fast after 22.00 hours the night before the BMR measurement, and to transport themselves to the research centre in a vehicle or by bus. They were also asked to avoid exercise the day before testing. Using a ventilated-hood system (Omnical, Maastricht University, the Netherlands), BMR was measured for $45 \mathrm{~min}$ at an environmental temperature of $22-24^{\circ} \mathrm{C}$. To eliminate effects of subject habituation to the testing procedure, the respiratory measurements during the first $10 \mathrm{~min}$ were discarded, and the following $20 \mathrm{~min}$ were used to calculate BMR. The criterion for this chosen time interval was the reproducibility of the calculated $\mathrm{BMR}$ values, resulting in the last $15 \mathrm{~min}$ of the $45 \mathrm{~min}$ during measurement being excluded because the subjects became more restless at the end of the measurement.

Gas analyses were performed by dual paramagnetic $\mathrm{O}_{2}$ analysers and dual infrared $\mathrm{CO}_{2}$ analysers (type 1156, 1507, 1520; Servomex, Crowborough, Sussex, UK), similar to the analysis system described by Schoffelen et al. (1997). Respiratory gas measurements were corrected for standard temperature, pressure and dry conditions. The Weir equation (Weir, 1949) was used to convert $\mathrm{VO}_{2}$ and $\mathrm{VCO}_{2}$ values to $\mathrm{EE}$ values.

Alternately before or after each BMR measurement, the function of the ventilated-hood system was checked with methanol burning during $20 \mathrm{~min}$. The methanol burner has been set to burn $0.2 \mathrm{~g} / \mathrm{min}$, which is equivalent to the production of $150 \mathrm{ml} \mathrm{CO} / \mathrm{min}$ and the consumption of $225 \mathrm{ml} \mathrm{O} 2 / \mathrm{min}$. Since an error percentage is dependent on burn-rate the expression of the error limit in absolute $\mathrm{ml} / \mathrm{min}$ is preferred, hence the limit values of 7.5 and $11 \mathrm{ml} / \mathrm{min}$ for $\mathrm{CO}_{2}$ and $\mathrm{O}_{2}$ respectively $(5 \%$ of $150 \mathrm{ml}$ $\mathrm{CO}_{2}$ and $5 \%$ of $225 \mathrm{ml} \mathrm{O}_{2}$ ).

\section{Physical activity}

Habitual physical activity was registered during $3 \mathrm{~d}$ before each BMR measurement with a tri-axial accelerometer for movement registration (Tracmor; Philips Research, Eindhoven, the Netherlands). The accelerometer was an improved version (same principle, but smaller) of the accelerometer used in previous studies (Bouten et al. 1996). It measures accelerations in the anteroposterior, medio-lateral and vertical directions. The dimensions of the accelerometer are $72 \times 27 \times 8 \mathrm{~mm}$ and it weighs $22 \mathrm{~g}$. Subjects wore the accelerometer in a waist belt at the 
low back during waking hours and recorded the times when they got up, put the Tracmor on and off, and when they went to bed. The registered accelerations in counts per min were used as an objective measure for the physical activity level of each subject.

\section{Body mass and body composition}

Body weight was estimated directly after every BMR measurement as well as 1 week before the first BMR measurement. Subjects were weighed in underwear before any food consumption and after emptying the bladder, on a digital balance accurate to $0.01 \mathrm{~kg}$ (KCC 300; Mettler, Greifensee, Switzerland).

Measurements of body composition were performed twice, both after the first and the second BMR measurement. Body composition was estimated from the threecompartment model of Siri (1956). Underwater weighing with simultaneous assessment of residual lung volume was used to assess body density. The lung volume was measured with the He dilution technique using a spirometer (Volugraph 2000; Mijnhardt, Bunnik, the Netherlands). The ${ }^{2} \mathrm{H}$ dilution method was used to determine total body water according to the Maastricht protocol (Westerterp et al. 1995). Subjects had to drink a ${ }^{2} \mathrm{H}$ dilution $(70 \mathrm{~g}$ with an enrichment of 5 atom \% excess ${ }^{2} \mathrm{H}$ ) in the evening before the measurements after the collection of a baseline urine sample. The next morning after a $10 \mathrm{~h}$ equilibration period a second urine sample was collected.

\section{Statistics}

Data are presented as mean values and standard deviations.

A one-way factor ANOVA for repeated measures was used to compare the three measurements of BMR, physical activity and body weight. The factor sex was taken as a between-subject variable.

Furthermore the coefficient of variation, the mean differences between the measurements and the coefficient of correlation were used to compare the BMR values between the three visits for each subject.

To assess the contribution of independent variables to the intra- and inter-variability of BMR measurements multiple and simple linear regression analyses were used.

The SPSS program, version 10 (SPSS Inc., Chicago, IL), was used for the statistical analysis.

\section{Results}

Before the statistical analysis was performed eight measurements were corrected for within-machine variability larger than $7.5 \mathrm{ml} / \mathrm{min}$ for $\mathrm{CO}_{2}$ or $11 \mathrm{ml} / \mathrm{min}$ for $\mathrm{O}_{2}$ . Furthermore five measurements were excluded from the analysis because subjects admitted their non-compliance to the protocol in relation to fasting. Mean measurements of BMR are presented in Table 2. There was no evidence of significant differences in BMR between the three measurements and the variation was not different for men and women (ANOVA repeated measures, $P=0 \cdot 88$ ). The mean intra-individual $\mathrm{CV}$ was 3.3 (SD $2 \cdot 1) \%$. The $\mathrm{CV}$ of BMR ranged from 0.4 to $7.2 \%$. The mean difference between the three measurements was $0.3 \quad(\mathrm{SD} 0.3) \quad \mathrm{MJ} / 24 \mathrm{~h}$ (between measurement 1 and 2), $0.2(\mathrm{SD} 0 \cdot 2$ ) $\mathrm{MJ} / 24 \mathrm{~h}$ (between measurement 2 and 3) and $0.3(\mathrm{SD} 0 \cdot 2$ ) $\mathrm{MJ} / 24 \mathrm{~h}$ (between measurement 1 and 3) (Table 3). The coefficient of correlation was 0.939 between measurement 1 and 2, 0.918 between measurement 1 and 3 and 0.980 between measurement 2 and 3.

Physical activity tended to be lower the last day before measurement 2 (8406 (SD 3308) kcounts/min) in comparison with the last day before measurement 1 (9599 (SD 4695) kcounts/min) and measurement 3 (9204 (SD 4091) kcounts/ min) (Table 2) but the differences were not significant and the variation was the same for men and women (ANOVA repeated measures, $P=0 \cdot 21$ ). The physical activity on day 3 and day 2 before the BMR measurement is presented in Table 2 and is similar to the results of the last day before testing (Table 2). The mean intra-individual CV for physical activity was 21 (SD 18) \% and it ranged from 0 to $65 \%$. Differences in body weight between the measurements seemed small (Table 2), but were significant. The variation in body weight did not depend on the factor sex (ANOVA repeated measures, $P<0.01$ ).

Differences between BMR could not be explained by differences in physical activity the day before testing (simple regression analysis) or by changes in body mass (simple regression analysis).

The mean inter-individual $\mathrm{CV}$ of BMR measurements was 18.0 (SD 1.6) \% and thus much larger than the intraindividual $\mathrm{CV}$ of BMR measurements. Most of this variation was explained by fat-free mass differences between the subjects $\left(r^{2} 0 \cdot 76, P<0 \cdot 001\right.$; simple regression analysis). Other variables, including physical activity the

Table 2. Basal metabolic rate and physical activity measured repeatedly at 2-week intervals in nineteen adults* (Mean values and standard deviations)

\begin{tabular}{|c|c|c|c|c|c|c|}
\hline & \multicolumn{2}{|c|}{ Measurement 1} & \multicolumn{2}{|c|}{ Measurement 2} & \multicolumn{2}{|c|}{ Measurement 3} \\
\hline & Mean & SD & Mean & SD & Mean & SD \\
\hline BMR (MJ/24h) & 6.59 & $1 \cdot 16$ & $6 \cdot 86$ & $1 \cdot 36$ & $6 \cdot 87$ & $1 \cdot 15$ \\
\hline Body weight $(\mathrm{kg})$ & 68.6 & $13 \cdot 2$ & 68.9 & $13 \cdot 3$ & $69 \cdot 5$ & 12.9 \\
\hline Physical activity day 1 (kcounts/min) & 10288 & 6790 & 9346 & 3587 & 9565 & 2714 \\
\hline Physical activity day 2 (kcounts/min) & 10469 & 5539 & 9706 & 2676 & 9285 & 3137 \\
\hline Physical activity day 3 (kcounts/min) & 9599 & 4695 & 8406 & 3308 & 9204 & 4091 \\
\hline
\end{tabular}

${ }^{*}$ For details of subjects and procedures, see Table 1 and p. 420. 
Table 3. Coefficient of variation of intra-individual variance in basal metabolic rate and the mean differences between the basal metabolic rate measurements*

(Mean values and standard deviations)

\begin{tabular}{|c|c|c|}
\hline & Mean & SD \\
\hline CV (\%) & $3 \cdot 3$ & $2 \cdot 1$ \\
\hline $\begin{array}{l}\text { Difference between measurement } 1 \text { and } 2 \\
(\mathrm{MJ} / 24 \mathrm{~h})\end{array}$ & 0.3 & 0.3 \\
\hline $\begin{array}{l}\text { Difference between measurement } 2 \text { and } 3 \\
(\mathrm{MJ} / 24 \mathrm{~h})\end{array}$ & 0.2 & 0.2 \\
\hline $\begin{array}{l}\text { Difference between measurement } 1 \text { and } 3 \\
(\mathrm{MJ} / 24 \mathrm{~h})\end{array}$ & 0.3 & 0.2 \\
\hline $\begin{array}{l}\text { Coefficient of correlation between measurement } 1 \\
\text { and } 2\end{array}$ & 0.939 & \\
\hline $\begin{array}{l}\text { Coefficient of correlation between measurement } 2 \\
\text { and } 3\end{array}$ & 0.918 & \\
\hline $\begin{array}{l}\text { Coefficient of correlation between measurement } 1 \\
\text { and } 3\end{array}$ & 0.980 & \\
\hline
\end{tabular}

*For details of subjects and procedures, see Table 1 and p. 420.

day before testing, did not explain any additional variation in BMR after adjustment for fat-free mass.

\section{Discussion}

The intra-individual $\mathrm{CV}$ in the present study was $3 \cdot 3$ (SD 2.1 ) \% with a range from 0.4 to $7.2 \%$. Similar results were observed by others (Table 4; Murgatroyd et al. 1987; Fredrix et al. 1990; Rieper et al. 1993; Figueroa-Colon et al. 1996; Ventham \& Reilly, 1999). Discrepancies could be due to differences in protocol, methodology and study population. The present study quantified potential factors affecting the reproducibility of BMR values, including the habitual physical activity the day before the measurement and non-compliance to fasting.

The measured BMR values were corrected when the subjects appeared not to be in a fasting state and when the within-machine variability was larger than $7.5 \mathrm{ml} / \mathrm{min}$ for $\mathrm{CO}_{2}$ or $11 \mathrm{ml} / \mathrm{min}$ for $\mathrm{O}_{2}$. The correction for the within-machine variability reduced the $\mathrm{CV}$ from 5.7 to $5 \cdot 2 \%$. After exclusion of measurements because of noncompliance to the protocol including fasting the $\mathrm{CV}$ decreased from $5 \cdot 2$ to $3 \cdot 3 \%$. Other authors do not describe these corrections mentioned and this could therefore partly explain the relatively high reproducibility found in the present study.

Differences between the three BMR measurements could not be explained by differences in physical activity the day before testing and by changes in body mass. Subjects were

Table 4. Mean intra-individual coefficients of variation for basal metabolic rate measurements as observed in earlier studies

\begin{tabular}{lc}
\hline & CV (\%) \\
\hline Rieper et al. (1993) & 4.3 \\
Figueroa-Colon et al. (1996) & 5.8 \\
Murgatroyd et al. (1987) & 5.9 \\
Ventham \& Reilly (1999) & $2 \cdot 6$ \\
Fredrix et al. (1990) & 3.3 \\
\hline
\end{tabular}

asked to avoid exercise the day before the measurements, but they were not limited in their habitual non-exercise activity. Physical activity the day before testing tended to be lower the day before measurement 2 (8406 (SD 3308) kcounts/min) in comparison with the day before measurement 1 (9599 (SD 4695) kcounts/min) and measurement 3 (9204 (SD 4091) kcounts/min), but the differences between the periods were not significant despite the high intra-individual variability (mean CV 21.5 (SD 18.7) \%).

Earlier studies were not conclusive about the effect of physical activity on BMR measurements (FreedmanAkabas et al. 1985; Maehlum et al. 1986; Weststrate \& Hautvast, 1990; Weststrate et al. 1990; Bahr et al. 1992; Herring et al. 1992; Melby et al. 1993; Smith \& McNaughton, 1993; Quinn \& Vroman, 1994; Williamson \& Kirwan, 1997; Almuzaini et al. 1998; Osterberg \& Melby, 2000; Melanson et al. 2002). Possible explanations for the discrepant results could be differences in the time interval between exercise and BMR measurement as well as variation in duration and intensity of the exercise. The results of several studies suggest that the magnitude of the elevation of EE above resting values during recovery from exercise primarily depends on the intensity of exercise and to a lesser extent on exercise duration (Sedlock et al. 1989; Gore \& Withers, 1990; Bahr et al. 1992; Smith \& McNaughton, 1993; Quinn \& Vroman, 1994; Borsheim et al. 1998). On the other hand, Melanson et al. (2002) found that EE immediately post exercise was elevated after low- and high-intensity exercise compared with no exercise, but they did not find differences in EE between low- and high-intensity exercise during the remainder of the day (Melanson et al. 2002).

Since, in the present study, only non-exercise physical activity was allowed, while the studies mentioned earlier estimated the effect of exercise, an evident influence of variation in non-exercise physical activity was not expected. Thus, in the present study no evidence is found for an important influence of the intra-individual variability of non-exercise activity on the reproducibility of BMR measurements.

The inter-individual $\mathrm{CV}$ of BMR in the present study was 18.0 (SD 1.6) \%, much larger than the intra-individual CV of BMR measurements. Most of this variation was explained by fat-free mass differences between the subjects $\left(r^{2} 0 \cdot 76\right.$, $P<0.001$; simple regression analysis). Other variables including physical activity did not explain any additional variation in BMR after adjustment for fat-free mass. This is in agreement with studies in which the fat-free mass or the active cell mass was shown to be the best predictor of BMR (Cunningham, 1980, 1991; Welle \& Nair, 1990; Goran \& Nagy, 1996; Lührmann et al. 2001).

In conclusion, the present study showed that BMR measurements as performed with a standard out-patient protocol result in highly reproducible BMR values; specifically, if corrected for non-compliance to the protocol including fasting and for within-machine variability larger than $7.5 \mathrm{ml} / \mathrm{min}$ for $\mathrm{CO}_{2}$ or $11 \mathrm{ml} / \mathrm{min}$ for $\mathrm{O}_{2}$. The lack of supervision of the fasting state is proven to be an evident disadvantage, while in the present study five measurements were excluded because of non-compliance to the fasting state. To assume that subjects will behave 
according to the rules of a protocol can therefore result in errors. Consequently it is important to exclude the measurement when subjects admit their non-compliance or in the case of an elevated respiratory quotient. The actual recommendation to refrain from exercise is sufficient to ensure accurate measurements of BMR in a repeated-measures design, while the BMR measurements in the present study were highly reproducible despite the within-subject variability in habitual physical activity. Finally, the correction for the within-machine variability improved the reproducibility and therefore a regular function check is recommendable. The present study proved that it is practically possible to check the function of the ventilated-hood system on a daily basis. On the other hand, this method is somewhat inconvenient and only required if the highest degree of resolution is needed. A weekly methanol calibration seems therefore a reasonable recommendation.

\section{References}

Almuzaini KS, Potteiger JA \& Gree SB (1998) Effects of split exercise sessions on excess postexercise oxygen consumption and resting metabolic rate. Can J Appl Physiol 23, 433-443.

Bahr R, Gronnerod O \& Sejersted OM (1992) Effect of supramaximal exercise on excess postexercise $\mathrm{O}_{2}$ consumption. Med Sci Sports Exerc 24, 66-71.

Borsheim E, Knardahl S, Hostmark AT \& Bahr R (1998) Adrenergic control of post-exercise metabolism. Acta Physiol Scand 162, 313-323.

Bouten C, Verboeket-van de Venne W, Westerterp K, Verduin M \& Janssen J (1996) Daily physical activity assessment: comparison between movement registration and doubly labelled water. J Appl Physiol 81, 1019-1026.

Cunningham JJ (1980) A reanalysis of the factors influencing basal metabolic rate in normal adults. Am J Clin Nutr 33, 2372-2374.

Cunningham JJ (1991) Body composition as a determinant of energy expenditure: a synthetic review and a proposed general prediction equation. Am J Clin Nutr 54, 963-969.

Figueroa-Colon R, Franklin FA, Goran MI, Lee JY \& Weinsier RL (1996) Reproducibility of measurement of resting energy expenditure in prepubertal girls. Am J Clin Nutr 64, 533-536.

Fredrix EWHM, Soeters PB, von Meyenfeldt MF \& Saris WHM (1990) Measurement of resting energy expenditure in a clinical setting. Clin Nutr 9, 299-340.

Freedman-Akabas S, Colt E, Kissilef HR \& Pi-Sunyer FX (1985) Lack of sustained increase in $\mathrm{VO}_{2}$ following exercise in fit and unfit subjects. Am J Clin Nutr 41, 545-549.

Goran MI \& Nagy TR (1996) Effect of the pre-testing environment on measurement of metabolic rate in children. Int J Obes Relat Metab Disord 20, 83-87.

Gore CJ \& Withers RT (1990) Effect of exercise intensity and duration on postexercise metabolism. J Appl Physiol 68, $2362-2368$.

Herring JL, Mole PA, Meredith CN \& Stern JS (1992) Effect of suspending exercise training on resting metabolic rate in women. Med Sci Sports Exerc 24, 59-65.

Lührmann PM, Herbert BM \& Neuhäuser-Berthold M (2001) Effects of fat mass and body fat distribution on resting metabolic rate in the elderly. Metabolism 50, 972-975.

Maehlum S, Grandmontangne M, Newsholme EA \& Sejersted OM (1986) Magnitude and duration of excess postexercise oxygen consumption in healthy young subjects. Metabolism $\mathbf{3 5}$, 425-429.

Melanson EL, Sharp TA, Seagle HM, et al. (2002) Effect of exercise intensity on 24-h energy expenditure and nutrition oxidation. J Appl Physiol 92, 1045-1052.

Melby C, Scholl C, Edward G \& Bullough R (1993) Effect of acute resistance exercise on postexercise energy expenditure and resting metabolic rate. $J$ Appl Physiol 75, 1847-1853.

Murgatroyd PR, Davies HL \& Prentice AM (1987) Intra-individual variability and measurement noise in estimates of energy expenditure by whole body indirect calorimetry. Br J Nutr $\mathbf{5 8}$, $347-356$.

Osterberg KL \& Melby CL (2000) Effect of acute resistance exercise on postexercise oxygen consumption and resting metabolic rate in young women. Int J Sport Nutr Exerc Metab 10, 71-81.

Quinn TJ \& Vroman NB (1994) Postexercise oxygen consumption in trained females: effect of exercise duration. Med Sci Sports Exerc 26, 908-913.

Rieper H, Karst H, Noack R \& Johnsen D (1993) Intra- and interindividual variations in energy expenditure of 14-15-year-old schoolgirls as determined by indirect calorimetry. Br J Nutr 69, 29-36.

Schoffelen PFM, Westerterp KR, Saris WHM \& ten Hoor F (1997) A dual-respiration chamber system with automated calibration. J Appl Physiol 83, 2064-2072.

Sedlock DA, Fissinger JA \& Melby CL (1989) Effect of exercise intensity and duration on postexercise intensity and duration on postexercise energy expenditure. Med Sci Sports Exerc 21, 662-666.

Siri WE (1956) The gross composition of the body. In Advances in Biological and Medical Physics, vol. IV, pp. 239-280 [JH Lawrence and CA Tobias, editors]. New York, NY: Academic Press.

Smith J \& McNaughton L (1993) The effects of intensity of exercise on excess postexercise oxygen consumption and energy expenditure in moderately trained men and women. Eur J Appl Physiol 67, 420-425.

Turley KR, McBride PJ \& Wilmore JH (1993) Resting metabolic rate measured after subjects spent the night at home vs. at a clinic. Am J Clin Nutr 58, 141-144.

Ventham JC \& Reilly JJ (1999) Reproducibility of resting metabolic rate measurement in children. $\mathrm{Br} J$ Nutr $\mathbf{8 1}$, 435-437.

Weir JB (1949) New methods for calculating metabolic rate with special reference to protein metabolism. J Physiol 109, $1-9$.

Welle S \& Nair KS (1990) Relationship of resting metabolic rate to body composition and protein turnover. Am J Physiol 258, E990-E998.

Westerterp KR, Wouters L \& van Marken Lichtenbelt WD (1995) The Maastricht protocol for the measurements of body composition and energy expenditure with labeled water. Obes Res 3, 49-57.

Weststrate JA \& Hautvast GAJ (1990) The effects of short-term carbohydrate overfeeding and prior exercise on resting metabolic rate ad diet-induced thermogenesis. Metabolism 39, $1232-1239$

Weststrate JA, Weys P, Poortvliet E, Deurenberg P \& Hautvast JGAJ (1990) Lack of a systematic sustained effect of prolonged exercise bouts on resting metabolic rate in fasting subjects. Eur J Clin Nutr 44, 91-97.

Williamson DL \& Kirwan JP (1997) A single bout of concentric resistance exercise increases basal metabolic rate 48 hours after exercise in healthy 59-77-year-old men. J Gerontol 52A, M352-M355. 